\title{
Twisted-angle-dependent Optical Behaviors of Intralayer Excitons and Trions in $\mathrm{WS}_{2} / \mathrm{WSe}_{2}$ Heterostructure
}

Jia Shi, ${ }^{1, \#}$ Yuanzheng Li ${ }^{1,2, \#}$ Zhepeng Zhang, ${ }^{3,4, \#}$ Weiqiang Feng, ${ }^{5}$ Qi Wang, ${ }^{1}$ Shuliang Ren, ${ }^{6,7}$ Jun Zhang, ${ }^{6,7}$ Wenna Du, ${ }^{1,8}$ Xianxin Wu, ${ }^{1,2}$ Xinyu Sui, ${ }^{1,2}$ Yang Mi, ${ }^{1}$ Rui Wang, ${ }^{1}$ Yuanhui Sun, ${ }^{6}$ Lijun Zhang, ${ }^{6}$ Xiaohui Qiu, ${ }^{1}$ Jiong Lu, ${ }^{9}$ Chao Shen, ${ }^{6,7^{*}}$ Yanfeng Zhang, ${ }^{4,5}$ Qing Zhang ${ }^{4 *}$ and Xinfeng Liu ${ }^{1 *}$

${ }^{1}$ CAS Key Laboratory of Standardization and Measurement for Nanotechnology, CAS Center for Excellence in Nanoscience, National Center for Nanoscience and Technology, Beijing 100190, P. R. China

${ }^{2}$ Centre for Advanced Optoelectronic Functional Materials Research and Key Laboratory of UV-Emitting Materials and Technology, Northeast Normal University, Ministry of Education, Changchun 130024, China

${ }^{3}$ Department of Materials Science and Engineering, College of Engineering, Peking University, Beijing 100871, China

${ }^{4}$ Academy for Advanced Interdisciplinary Studies, Peking University, Beijing 100871, P. R. China

${ }^{5}$ Key Laboratory of Automobile Materials of MOE, State Key Laboratory of Superhard Materials, and College of Materials Science and Engineering, Jilin University, Changchun 130012, China

${ }^{6}$ College of Materials Science and Opto-Electronic Technology, University of Chinese Academy of Science, Beijing 100049, China

${ }^{7}$ State Key Laboratory of Superlattices and Microstructures, Institute of Semiconductors, Chinese Academy of Sciences, Beijing 100083, China

${ }^{8}$ Key Laboratory of Semiconductor Materials Science, Beijing Key Laboratory of Low Dimensional Semiconductor Materials and Devices, Institute of Semiconductors, Chinese Academy of Sciences, Beijing, 100083, China

${ }^{9}$ Department of Chemistry Faculty of Science, National University of Singapore, 119077, Singapore 


\section{Contents}

2 S1. Power dependent PL spectra of monolayer $\mathrm{WSe}_{2}$

3 S2. SHG spectra of heterostructure with twisted angle of $60^{\circ}$

$4 \quad$ S3. Band structure of heterostructures with different twisted angles

5 S4. Power dependent PL spectra of heterostructures with different twisted angles

6 S5. Peak position versus excitation power of fitted exciton and trion in $\mathrm{WS}_{2} / \mathrm{WSe}_{2}$

7 S6. Low-frequency (LF) Raman spectra in $\mathrm{WS}_{2} / \mathrm{WSe}_{2}$

$8 \quad$ S7. Representative PL spectra of $\mathrm{WS}_{2} / \mathrm{WSe}_{2}$ heterostructure

9 S8. Temperature dependent PL spectra for heterostructure

10 S9. The instrument response function (IRF) for transient absorption

11 S10. Differential reflection signal measured from monolayer $\mathrm{WSe}_{2}$

$12 \mathrm{~S} 11$. Differential reflection signal measured from $\mathrm{WS}_{2} / \mathrm{WSe}_{2}$

13 S12. Carrier concentration calculated by the mass action model 
We performed a comparative study of the power dependence from the excitons, trion of $\mathrm{WSe}_{2}$. In Figure S1a-b, typical PL spectra of monolayer $\mathrm{WSe}_{2}$ and $\mathrm{WS}_{2}$ are exhibited, respectively. Figure S1c shows PL spectrum of monolayer $\mathrm{WSe}_{2}$ under different excitation power, and the spectrum is fitted as neutral exciton and negative charged trion by using Gauss function. As

6 exhibited in Figure S1d, the neutral exciton (solid blue circle) and trion (open pink circle)

7 show a linear dependence as a function of power. The results indicate that the range of power

8 applied does not give rise to the threshold of non-linear interaction, within which we can

9 guarantee the intrinsic proportional distribution of exciton and trion in the linear relation interval of material.
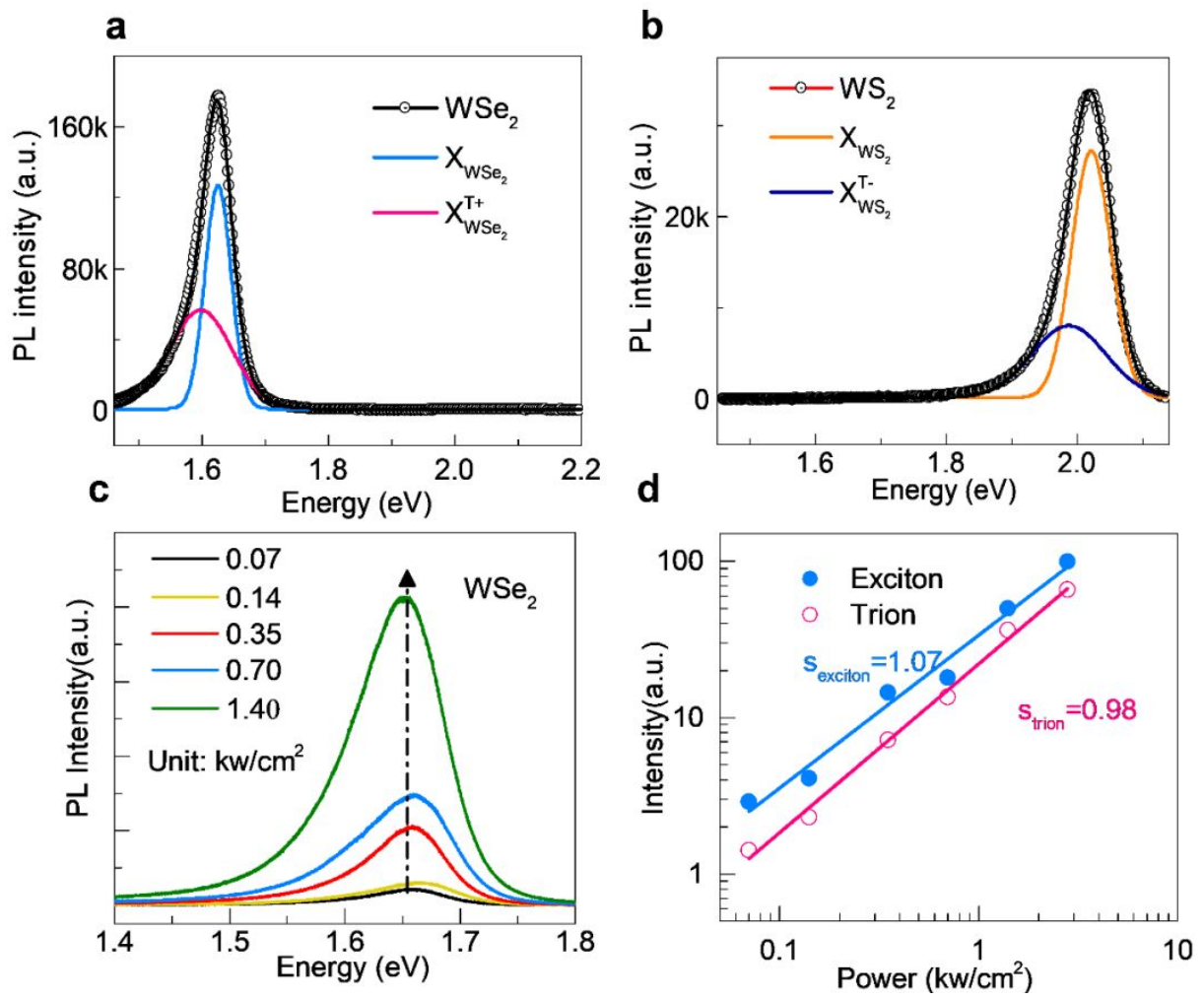

FigureS1 (a)-(b), typical PL spectra of monolayer $\mathrm{WSe}_{2}$ and $\mathrm{WS}_{2}$ are exhibited, respectively. (c) Power dependent PL spectrum of monolayer $\mathrm{WSe}_{2}$ where the wavelength of excitation beam is fixed at $514 \mathrm{~nm}$ with the power ranging from $50 \mu \mathrm{w}$ to $1 \mathrm{mw}$. (d) Normalized exciton (solid blue circle) and trion (open pink circle) emission intensity of monolayer $\mathrm{WSe}_{2}$ as a function of incident power presenting linear response to the incident power in a log-log scale. The slope of fitting function for exciton and trion of $\mathrm{WSe}_{2}$ is $s_{\text {exciton }}=1.07, s_{\text {trion }}=0.98$, 


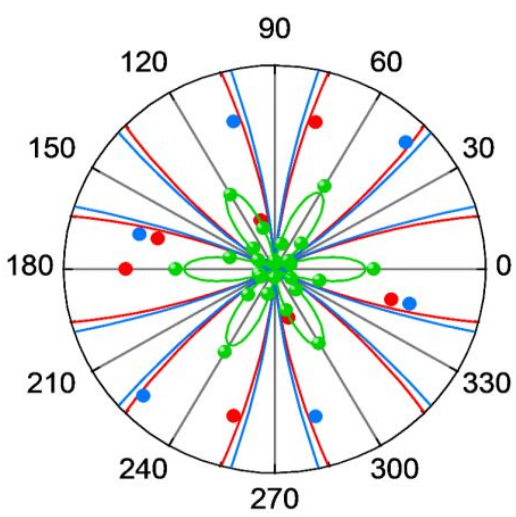

3 Figure S2. Zoom in- Angle dependence of SHG intensity for $\mathrm{WS}_{2} / \mathrm{WSe}_{2}$ heterostructure with 4 twisted angles of $60^{\circ}$ extracted in Figure 2f. 


\section{S3. Band structure of heterostructures with different twisted angles}

2 Figure S3a-c exhibits atomic schematic diagrams of heterostructure with twisted angle of 0, 30 3 and 60 degree from top view and front view, respectively. The band structures of

4 heterostructure with different twisted angle are shown in Figure S3d-f, in which the

5 heterostructure keeps the direct band-gap at the $K$ Point of the Brillouin zone. The energy band

6 structure shows the contribution from $\mathrm{WS}_{2}$ in the heterostructure in red and the contribution

7 from $\mathrm{WSe}_{2}$ in blue. Moreover, $\mathrm{CBM}$ is mainly determined by $\mathrm{WS}_{2}$ and $\mathrm{VBM}$ is mainly

8 determined by $\mathrm{WSe}_{2}$.

a

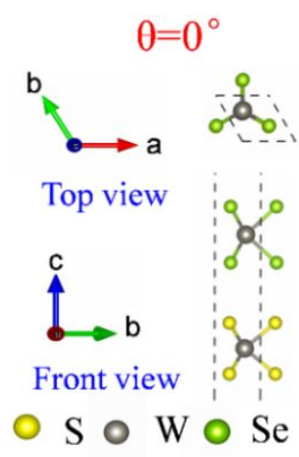

d

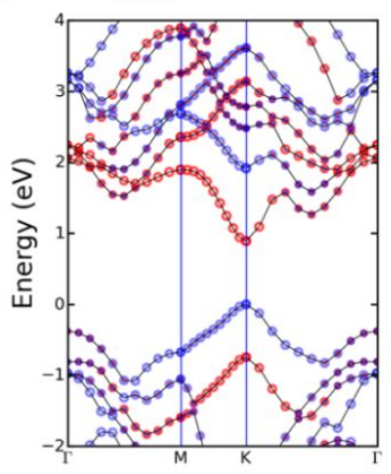

b

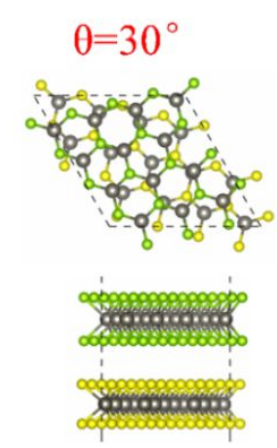

e

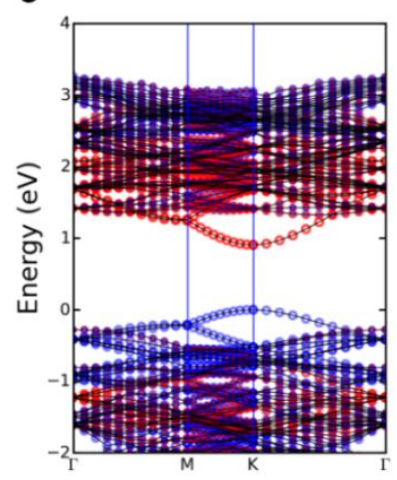

C

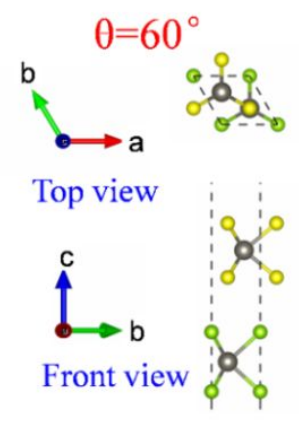

f

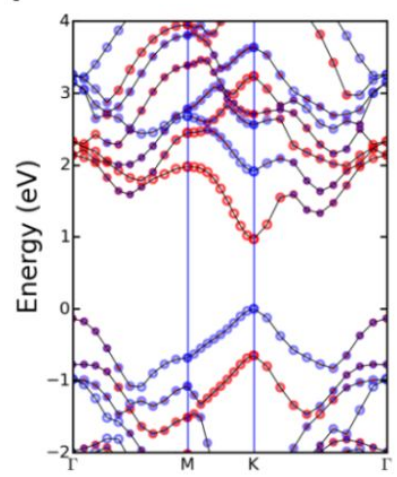

FigureS3. (a)-(c) Atomic schematic diagrams of heterostructure with twisted angle of 0 degree, 30 degree and 60 degree from top view and front view, respectively. (d)-(f) Band structures of 0-degree, 30-degree and 60-degree stacked heterostructure calculated by Density Functional Theory (DFT) method. 
S4. Power dependent PL spectra of heterostructures with different twisted angles
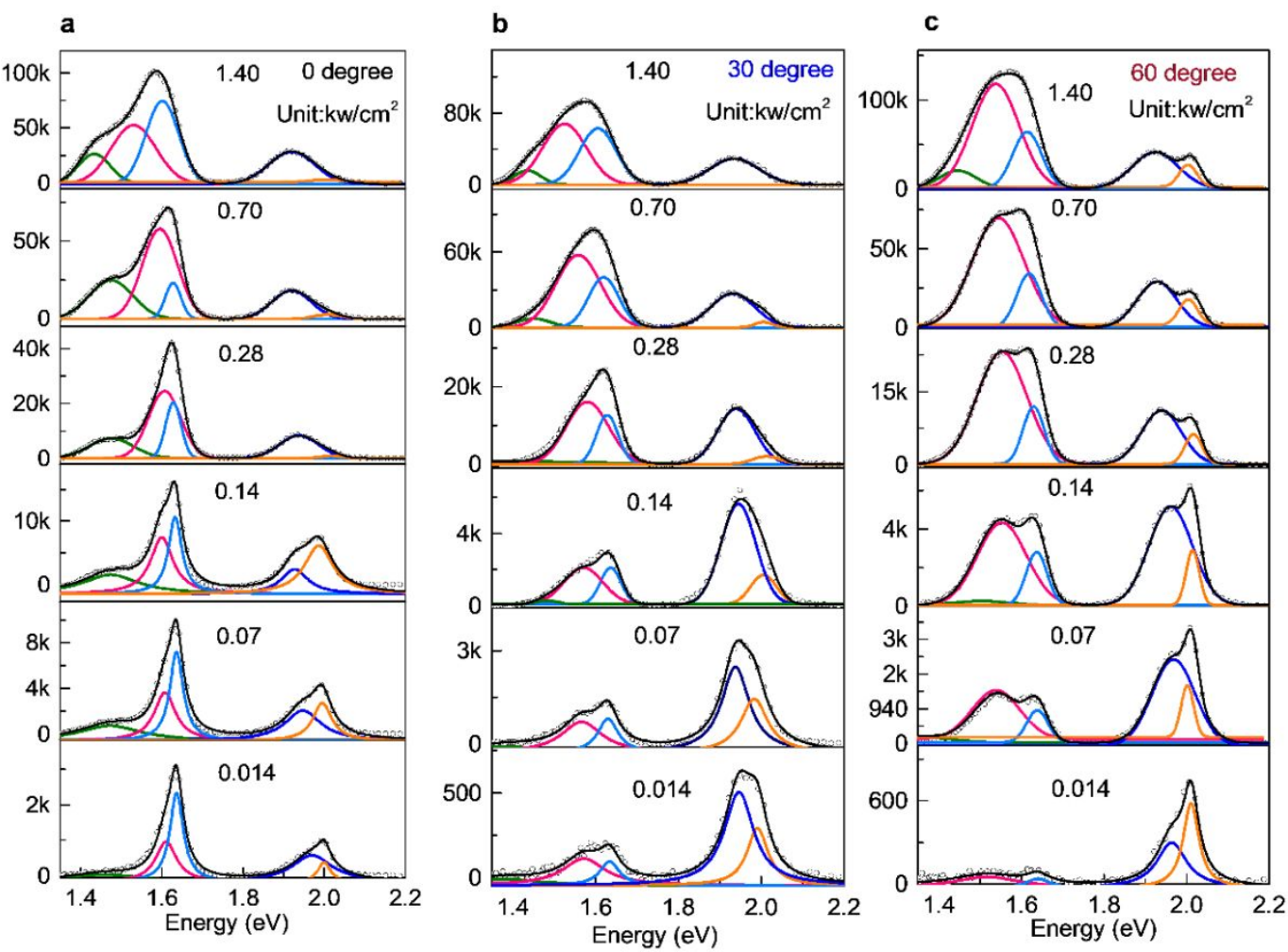

FigureS4 (a)-(c) Power dependent PL spectrums of $\mathrm{WS}_{2} / \mathrm{WSe}_{2}$ heterostructure with twisted angle of 0,30 and 60 degree. Green, pink and blue filled peaks represent interlayer exciton $\left(\mathrm{X}_{\mathrm{I}}\right)$ between $\mathrm{WS}_{2}$ and $\mathrm{WSe}_{2}$, positive trion $\mathrm{X}^{\mathrm{T}+}$ and neutral exciton $\mathrm{X}$ in $\mathrm{WSe}_{2}$, respectively. Mazarine and orange filled peaks are negative trion $\mathrm{X}^{\mathrm{T}-}$ and neutral exciton $\mathrm{X}$ in $\mathrm{WS}_{2}$. 
2 The energies of the exciton $\left(\mathrm{E}_{\mathrm{X}}\right)$ and trion peaks $\left(\mathrm{E}_{\mathrm{T}}\right)$ increased as we increase the excitation

3 level as shown in Figure S5. The energy difference between them called as trion binding

4 energy, is the energy required to convert the trion to an exciton and a free charge (electron or

5 hole). By increasing the excitation level, the Fermi surface which determines the release of free

6 charge increased. As a result, the trion binding energy increases with the Fermi surface when

$7 \quad$ increases with the excitation level.

a

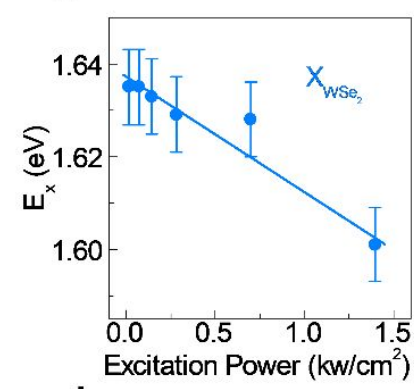

d

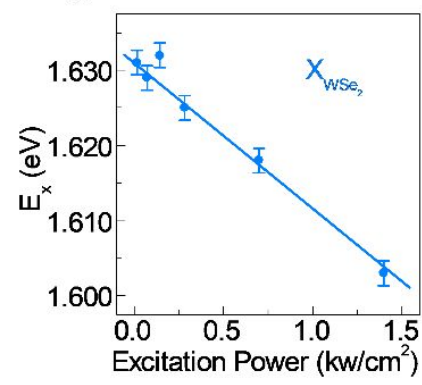

g

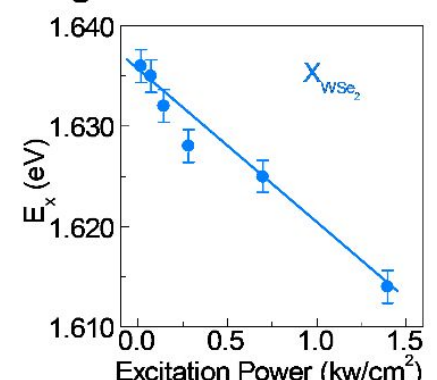

b
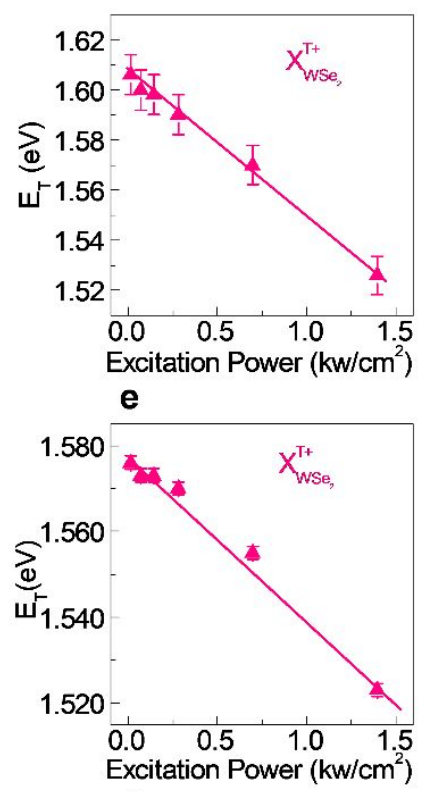

h

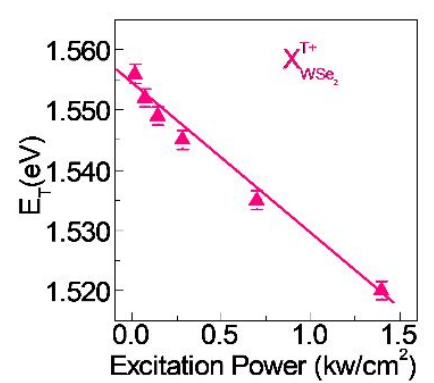

c
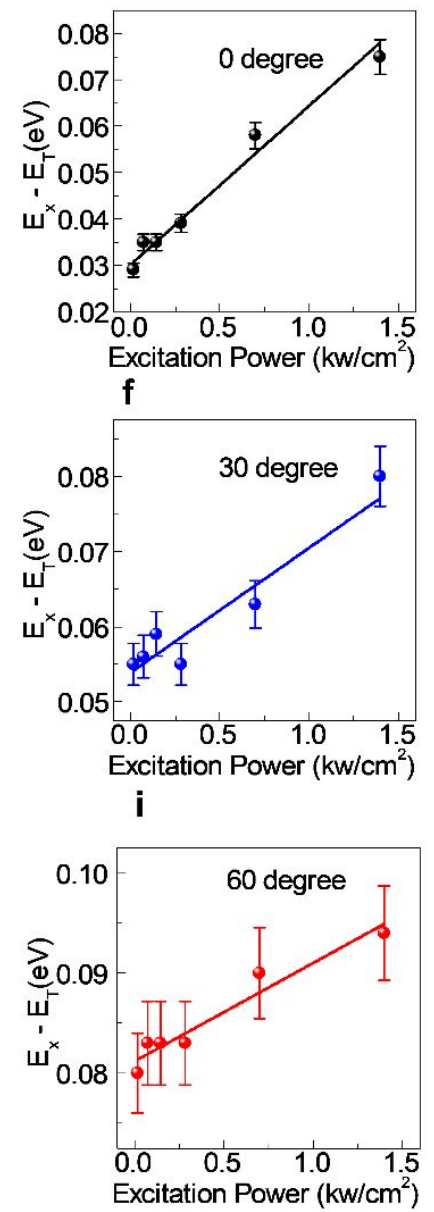

FigureS5 (a)-(i) Plot of peak position as a function of excitation power in $\mathrm{WS}_{2} / \mathrm{WSe}_{2}$ heterostructure with twisted angle of $0^{\circ}, 30^{\circ}$ and $60^{\circ}$, respectively. (a)(d)(g) and (b)(e)(h) represent the exciton and trion peak positions deduced from the fits shown in Figure S4 and the trion binding energy as a function of the excitation power are shown in (c)(f)(i). 
3 We firstly carried out the low-frequency (LF) Raman spectra to determine the strength of 4 interlayer coupling between $\mathrm{WS}_{2} / \mathrm{WSe}_{2}$ heterostructures with different twisted angles. As

5 shown in Figure S6-a, the low-frequency Raman spectra of monolayer $\mathrm{WS}_{2}, \mathrm{WSe}_{2}$ as well as

6 heterostructures with representative twisted angle $\left(0^{\circ}, 30^{\circ}, 60^{\circ}\right)$ were carried out at room

7 temperature by using $532 \mathrm{~nm}$ laser. The layer breathing mode (LBM) as shown in Figure

8 Figure S6-b mainly reflects the behavior of the interlayer phonon vibrations of the entire 2D

9 layers and is highly sensitive to the stacking orientations. ${ }^{1} \mathrm{WS}_{2} / \mathrm{WSe}_{2}$ heterostructure with twisted angle of $0^{\circ}$ and $60^{\circ}$ exhibit higher LBM peak intensity than that of $30^{\circ}$ stacked

11 heterostructure, which means a stronger interlayer coupling strength between layers with 12 atomically clean interface. ${ }^{2-3}$ As previous literature reported, ${ }^{4}$ the A exciton exhibit distinct binding energies $\mathrm{Eb}(\mathrm{e}-\mathrm{h})$ in the $\mathrm{MX}_{2}(\mathrm{M}=\mathrm{Mo}, \mathrm{W}$, and $\mathrm{X}=\mathrm{S}$, Se, etc.) materials with different stacking orders. Wei-Ting Hsu et al. also confirmed that the intralayer exciton binding energy for $\mathrm{AA}\left(0^{\circ}\right)$ is larger than that of $\mathrm{AB}\left(60^{\circ}\right)$ stacked heterostructure. ${ }^{5}$ The similar conclusion also fit for the $\mathrm{WS}_{2} / \mathrm{WSe}_{2}$ heterostructures with $\mathrm{AA}$ and $\mathrm{AB}$ stacking sequence owing to a net dipole pointing from the WSe2 to the WS2 layer in AA stacked heterostructure as shown in Figure S6-b.
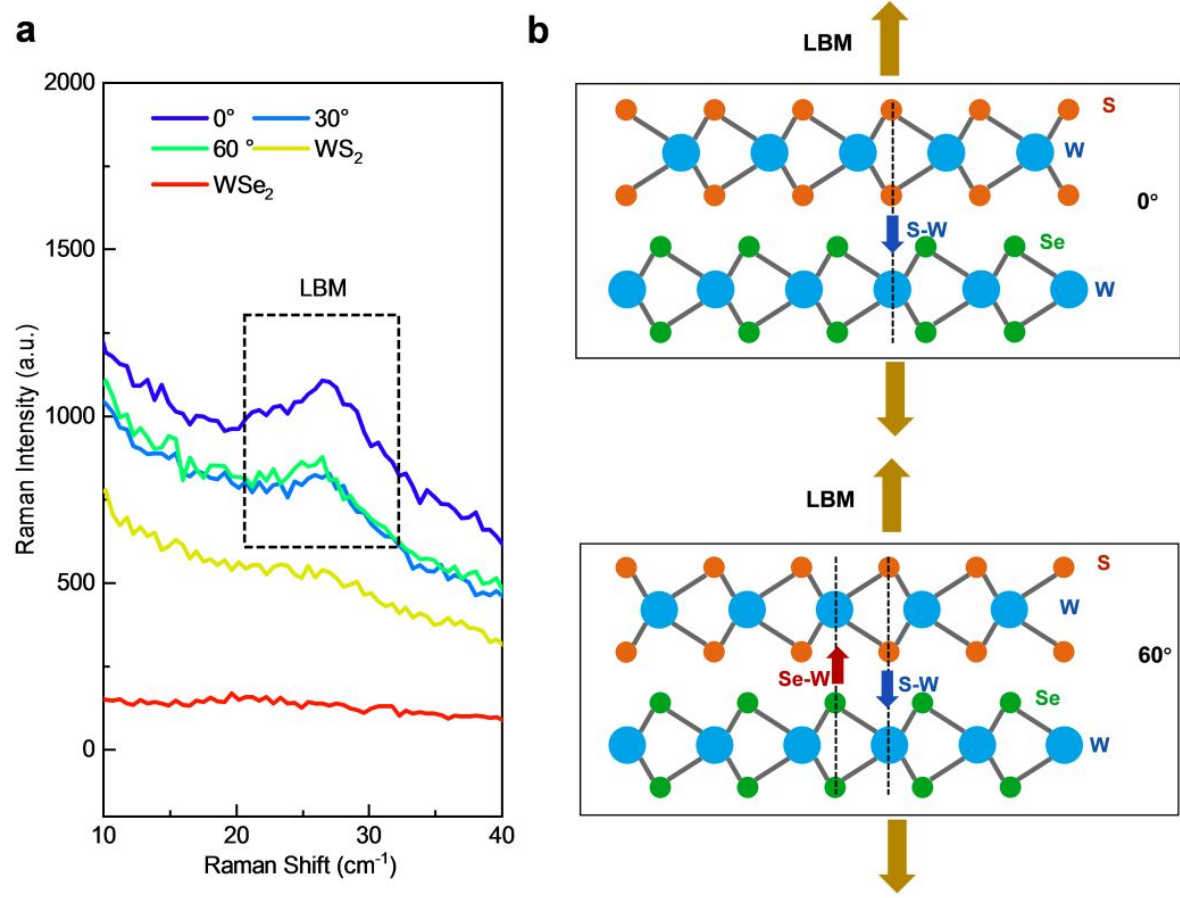
1 Figure S6 (a) Low-frequency Raman spectra of monolayer $\mathrm{WS}_{2}, \mathrm{WSe}_{2}$ and heterostructure 2 with twisted angle of $0^{\circ}, 30^{\circ}, 60^{\circ}$, respectively. (b) Schematics view of layer breathing mode 3 (LBM) vibrations for the $\mathrm{WS}_{2} / \mathrm{WSe}_{2}$ heterostructure with twisted angle of $0^{\circ}$ and $60^{\circ}$. 4 Sandybrown arrows represent the breathing mode between layers. Red and Blue arrows 5 represent the different type of Interlayer atomic registries of $0^{\circ}$ and $60^{\circ}$ stacking with $3 \mathrm{R}$-like 6 and $2 \mathrm{H}$-like stacking, respectively. 


\section{S7. Representative PL sepectra of $\mathrm{WS}_{2} / \mathrm{WSe}_{2}$ heterostructure}

2 We have done more PL spectra of $\mathrm{WS}_{2} / \mathrm{WSe}_{2}$ heterostructures with other twisted angles ranging from $3^{\circ}$ to $63^{\circ}$ and try to reveal the correlation between the twisted angles and the origin of the different optical behaviors. The corresponding PL spectra were exhibited in Figure S7-1 to S7-3 and fitted by the Gaussian function. As can be seen, the overall distribution of the peaks in the heterostructure with different twisted angles are basically consistent with the representative PL spectra of heterostructures with twisted angle of $0^{\circ}, 30^{\circ}$ and $60^{\circ}$ in the manuscript. For heterostructure with small twisted angle, interlayer excitons exhibit comparable PL Intensity with that of other peaks. In terms of the heterostructure with twisted angle between $10^{\circ}$ to $30^{\circ}$, positively charged trions of $\mathrm{WSe}_{2}$ started to occupy a comparable part of the PL emission. For the heterostructure with twisted angle ranging from $30^{\circ}$ to $60^{\circ}$, the ratio of trions in $\mathrm{WSe}_{2}$ exhibits gradual dominant trend and an obvious trion peak can be seen for the heterostructure with twist angle nearly $60^{\circ}$.

In this work, we have mainly studied the competition mechanism of quasi-particles including intralayer excitons, trions, and interlayer excitons in heterostructures with different twisted angles at room temperature. We have also supplemented several representative groups of PL spectra of $\mathrm{WS}_{2} / \mathrm{WSe}_{2}$ heterostructure with different twisted angles to prove the accuracy of the data. The corresponding PL spectra are shown in Figure S7-4a and it can be seen that the ratio of these quasi-particle in spectra conforms to the description in the manuscript fitted by Gaussian function. For $0^{\circ}$ stacked heterostructure, interlayer excion exhibits comparable PL intensity with that of other peaks. In terms of the heterostructure with twisted angle of $30^{\circ}$, positively charged trions of $\mathrm{WSe}_{2}$ occupy a relatively large part of the PL emission. For the heterostructure with twisted angle of $60^{\circ}$, the ratio of trions in $\mathrm{WSe}_{2}$ exhibits overwhelming predominance. Therefore, the repeatable experimental data allows us to obtain a histogram of the statistical distribution of the peak ratio, which also confirms that our statistical results are reliable. 

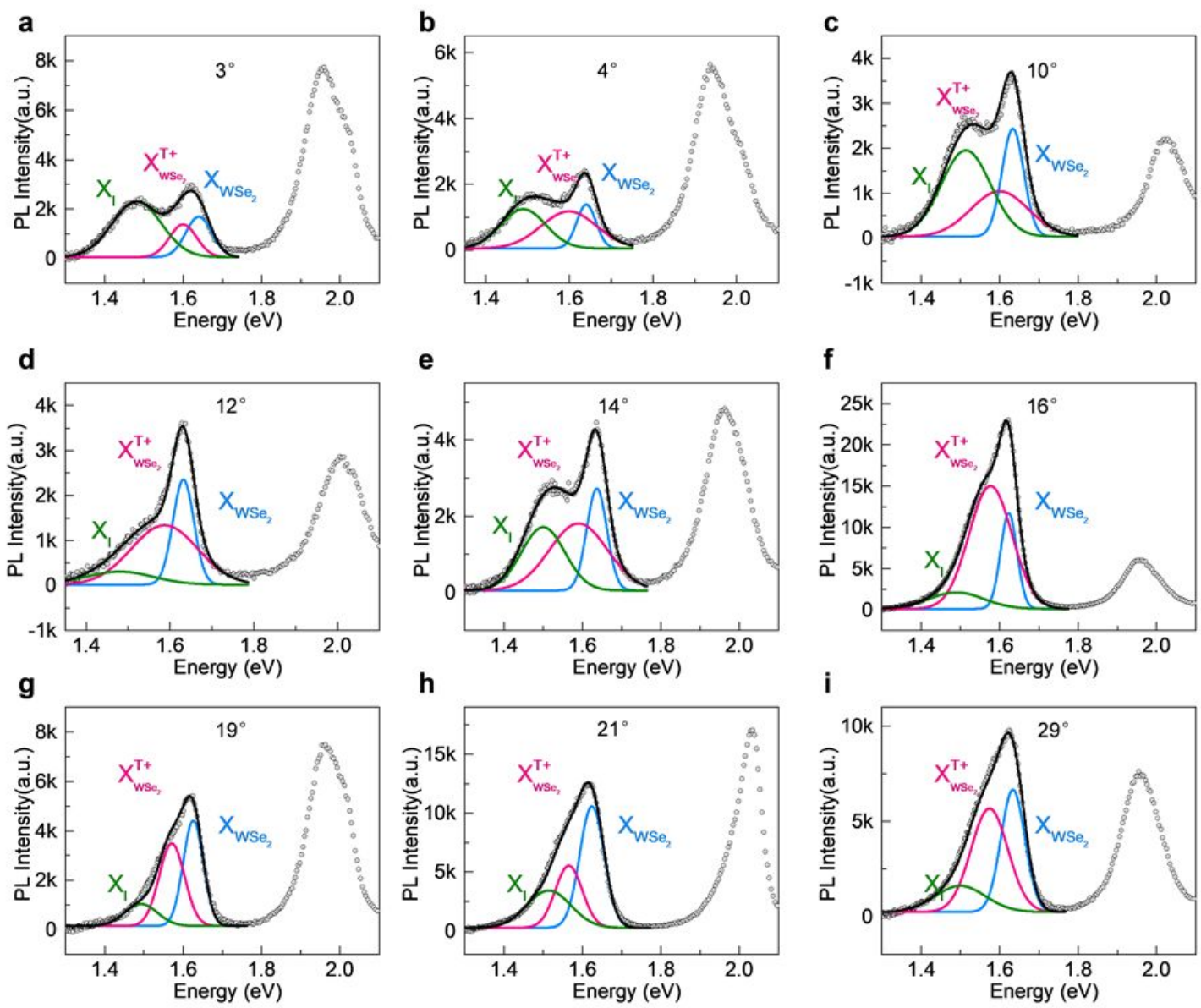

2 Figure S7-1. (a)-(i) Representative PL spectra of $\mathrm{WS}_{2} / \mathrm{WSe}_{2}$ heterostructure with twisted 3 angles ranging from $3^{\circ}$ to $29^{\circ}$, respectively. The spectral bands are fitted by a Gaussian 4 function to the observed spectral shapes. 

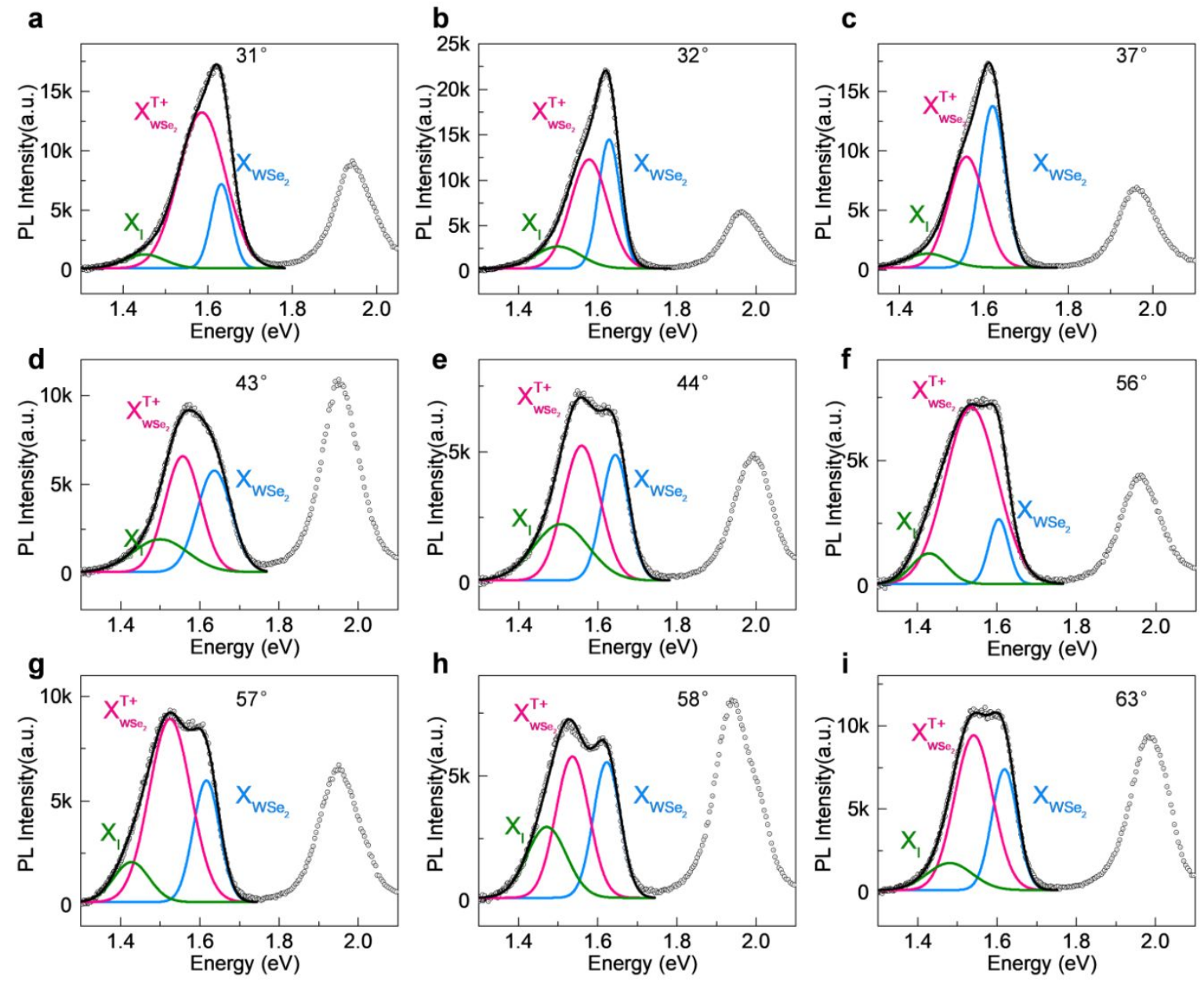

2 Figure S7-2. (a)-(i) Representative PL spectra of $\mathrm{WS}_{2} / \mathrm{WSe}_{2}$ heterostructure with twisted

3 angles ranging from $31^{\circ}$ to $63^{\circ}$, respectively. The spectral bands are fitted by a Gaussian 4 function to the observed spectral shapes. 

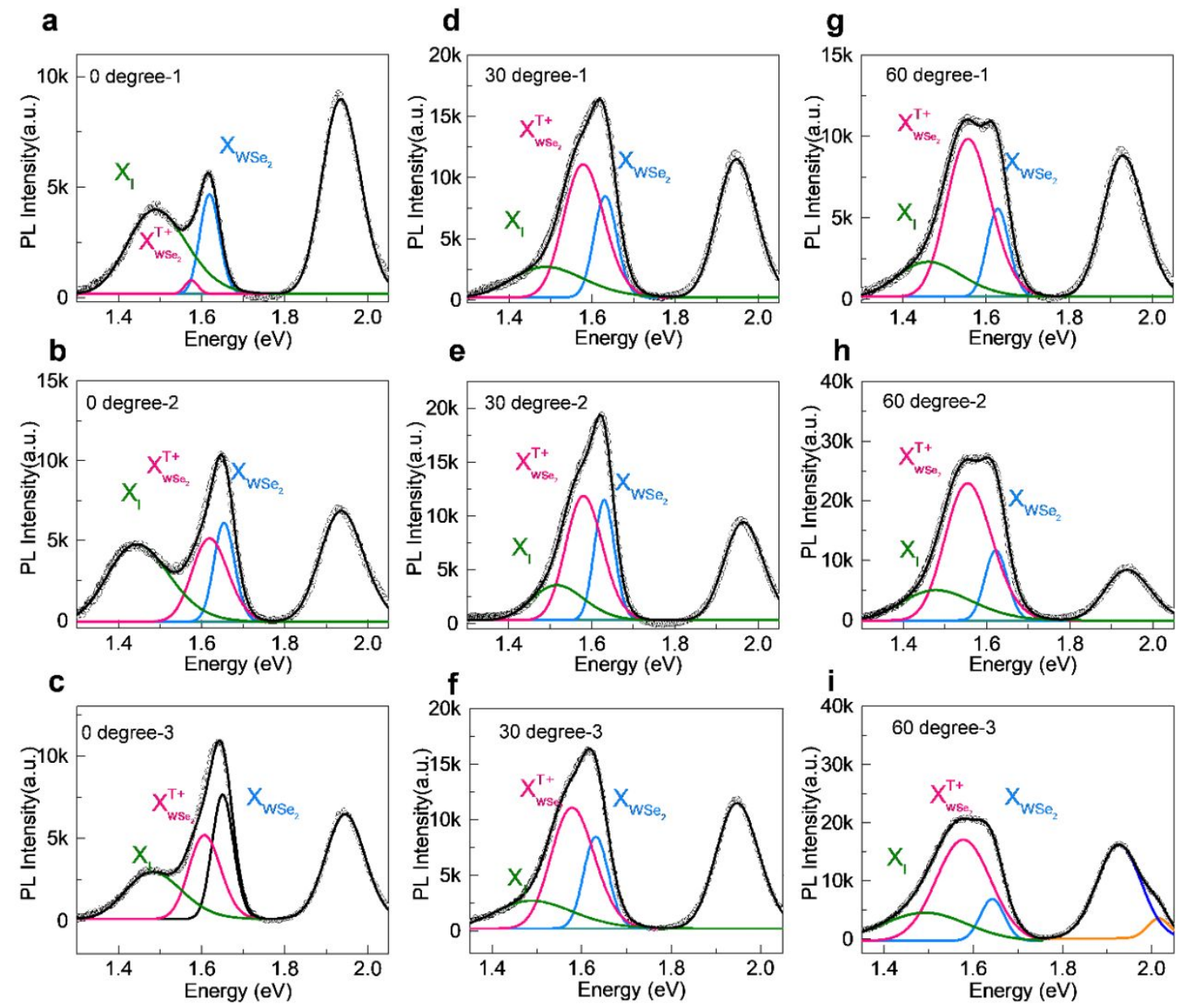

Figure S7-3. (a)-(i) Representative PL sepectra of $\mathrm{WS}_{2} / \mathrm{WSe}_{2}$ heterostructure with twisted angles of $0^{\circ}, 30^{\circ}$ and $60^{\circ}$, respectively. The spectral bands are fitted by a Gaussian function to

4 the observed spectral shapes.

The statistical histogram of different proportion of $\mathrm{XWSe}_{\mathrm{WS}_{2}}, \mathrm{X}_{\mathrm{WSe} 2}^{\mathrm{T}+}$ and $\mathrm{X}_{\mathrm{I}}$ as a function of twisted angle in $\mathrm{WS}_{2} / \mathrm{WSe}_{2}$ heterostructure are shown in Figure $\mathbf{S 7 - 4 b}$. For the heterostructure with small twist angle of $3^{\circ}$ or $4^{\circ}$, the interlayer excitons still occupy a large proportion owing to the momentum matching. For the heterostructure with twist angles ranging from nearly $10^{\circ}$ to $60^{\circ}$, the trions and excitons started to occupy a dominant proportion compared to the interlayer excitons. In order to reveal the competition between the trions and the neutral excitons in the heterostructure, we plot the PL intensity ratio of them as a fuction of twisted angle in Figure S7-4c. For heterostructure with twisted angle close to $0^{\circ}, 30^{\circ}$ and $60^{\circ}$, the intensity ratio of trions and excitons is basically the same as that reported in the manuscript, as

14 indicated by the red dashed line in the figure, that is, the ratio of trions begins to improve at $30^{\circ}$ and get a dominant proportion at $60^{\circ}$ heterostructure. For the other twisted angles, the overall 
1 ratio exhibit a similar increased trend as the angle increases as indicated by the black dashed

2 line in the figure.
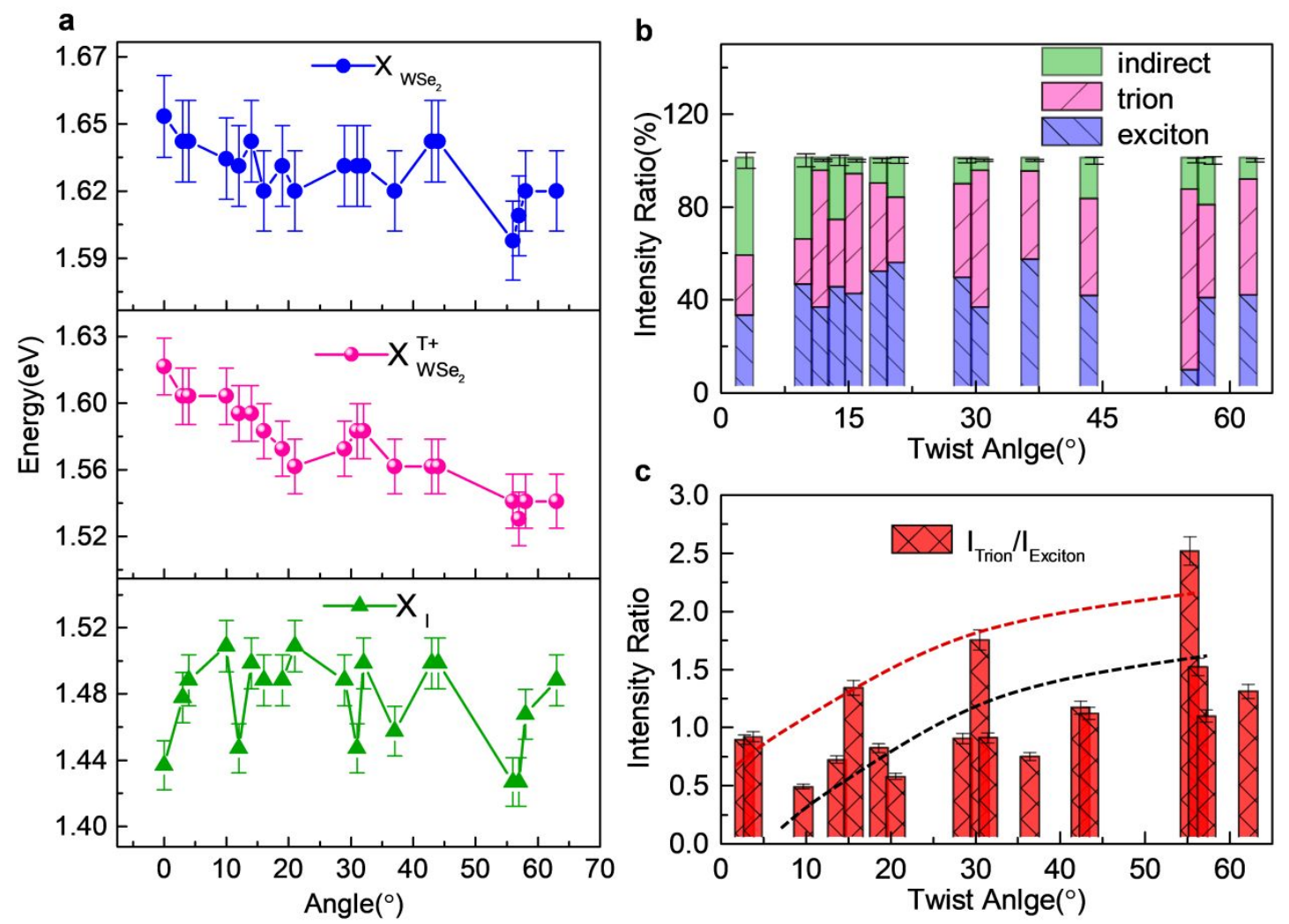

4 Figure S7-4. (a) Peak positions of neutral excitons $\left(\mathrm{X}_{\mathrm{WSe}_{2}}\right)$, positive trions $\left(\mathrm{X}_{\mathrm{WSe}^{2}}^{\mathrm{T}+}\right)$ in $\mathrm{WSe}_{2}$, 5 and interlayer exciton $\left(\mathrm{X}_{\mathrm{I}}\right)$ between layers as a function of twisted angle in $\mathrm{WS}_{2} / \mathrm{WSe}_{2}$ 6 heterostructure. (b) The statistical histogram of different proportion of $\mathrm{X}_{\mathrm{WSe}_{2}}, \mathrm{X}_{\mathrm{WSe} 2}^{\mathrm{T}+}$ and $\mathrm{X}_{\mathrm{I}}$ 7 as a function of twisted angle in $\mathrm{WS}_{2} / \mathrm{WSe}_{2}$ heterostructure. (c) Histogram of the PL intensity 8 ratio of trions and excitons in $\mathrm{WSe}_{2}$ as a function of twisted angle in $\mathrm{WS}_{2} / \mathrm{WSe}_{2}$ 9 heterostructure. The red and black dashed lines represent the trend of intensity distribution with 10 angle, respectively. 
2 Moreover, we measured low temperature (77K) PL sepectra of heterostructure with twisted 3 angle of $0^{\circ}, 30^{\circ}$ and $60^{\circ}$ to further confirm the assignment of PL peak fitting results in Figure

$4 \quad 2 \mathrm{~g}$. In our work, to ensure the diversity of twist angles in heterostructure, heterostructures were 5 obtained by random stacking of single-layer $\mathrm{WS}_{2}$ and $\mathrm{WSe}_{2}$ which were grown by CVD 6 method. Since the defect density in CVD-grown material is relatively larger than that in the 7 mechanically exfoliated sample, the defect may occupy the main PL ratio at the liquid helium 8 temperature $(4 \mathrm{~K})$, which is inconsistent with the original intention of our experiment. The 9 corresponding PL spectra ranging from room tempertature to liquid nitrogen temperature (77 $10 \mathrm{~K}$ ) of heterostructures with twisted angles of $0^{\circ}, 30^{\circ}, 60^{\circ}$ are shown in Figure S8. As can be 11 seen from Figure S8, as the temperature decreases, the proportion of luminescence of 12 interlayer excitons increases significantly in $0^{\circ}$ stacked heterostructure at low temperature. The heterostructure with twist angle of $0^{\circ}$ satisfies the angular momentum matching; the PL emission of interlayer excitons is more competitive than other quasi-particles at low temperature. The results help us confirm that the peak assignment for the interlayer excitons in heterostructure with twisted angle of $0^{\circ}$ in Figure $\mathbf{2 g}$ is reliable. In terms of heterostructure 17 with twist angle of $30^{\circ}$, similar to the case at room temperature, no obvious interlayer excitons are observed, and the luminescence intensity of $\mathrm{WSe}_{2}$ also decreases with the decrease of temperature. While the PL peaks of negatively charged trions and neutral excitons in $\mathrm{WS}_{2}$ are clearly separated and the binding energy of trions are approximately $40 \mathrm{meV}$. For $60^{\circ}$ stacked 21 heterostructure, negatively charged trions and neutral excitons in $\mathrm{WS}_{2}$ still dominate the PL emission intensity as the temperature goes down. The luminescence intensity of $\mathrm{WSe}_{2}$ is weaker than that of $\mathrm{WS}_{2}$, but the positively charged trions and neutral excitons of $\mathrm{WSe}_{2}$ are clearly separated. The binding energy of trions in $\mathrm{WSe}_{2}$ is calculated as around $90 \mathrm{meV}$ which is consistent with the results at room temperature in the manuscript and indicates that the peak fitting of trions are reliable at room temperature. 

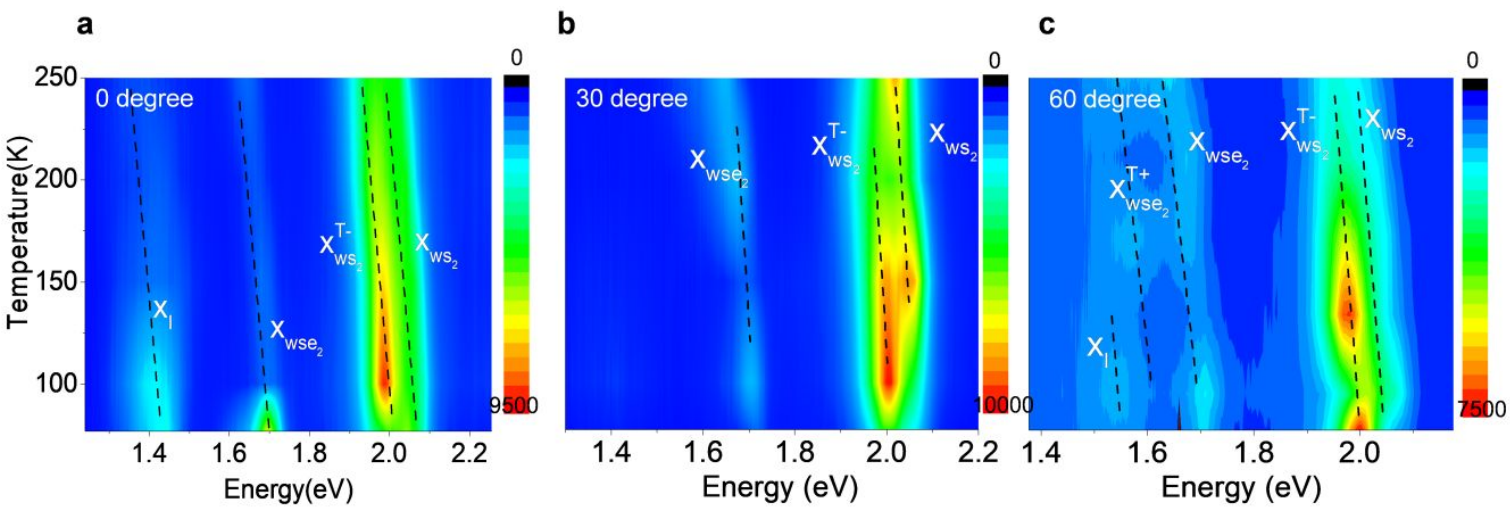

2 Figure S8. (a)-(c) Temperature dependent PL spectra for heterostructure with twist angle of $0^{\circ}$, $330^{\circ}$ and $60^{\circ}$, respectively. 
2 We measured the instrument response function (IRF) for transient absorption as shown in

3 Figure S8. In the experiment, quartz was taken as the standard sample to test the response

4 curve of the instrument. By fitting the curve, the time resolution of the instrument is up to 100

5 fs, which is less than the lifetime of carriers (200 fs) as mentioned in the manuscript. It is 6 confirmed that the lifetime data measured before is reliable.

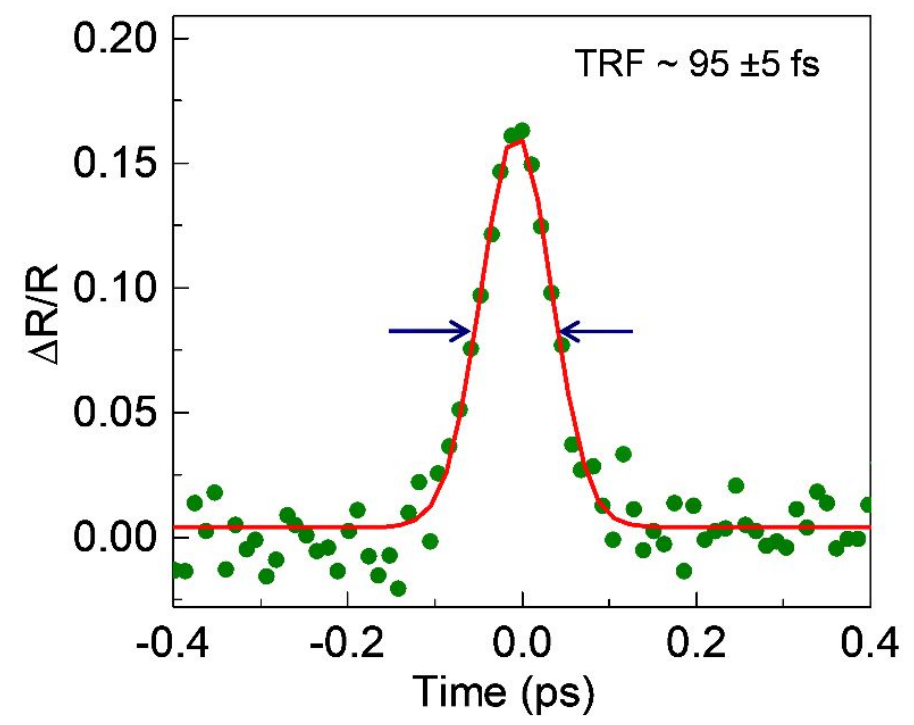

8 Figure S9. Typical curve of instrument response function (IRF), the time resolution is around 995 fs. 
2 Differential reflection signal measured from monolayer $\mathrm{WSe}_{2}$ as shown in Figure $\mathbf{S 1 0}$ are

3 excited by a $\sim 100 \mathrm{fs}$ pump pulse with a central wavelength of $580 \mathrm{~nm}$ and an energy fluence of

$4 \sim 1.9 \mu \mathrm{J} \mathrm{cm}^{-2}$. The photon energy is above the band gap of monolayer $\mathrm{WSe}_{2}$ and there appears

5 to be a negative component which can be induced by a rapid bandgap renormalization process.

6 For the neutral excitons of $\mathrm{WSe}_{2}$, we observed a rapid absorption process and attributed it to

7 two possible reasons. One is that the excitons quickly absorb excess holes preferentially form

8 trions, and the other is the process of rapid band renormalization process due to the

9 introduction of pump light. Table S1 exhibit the fast and slow decay time of both exciton and

10 trion for monolayer $\mathrm{WSe}_{2}$, where and the formation time of trion is around $3.2 \mathrm{ps,} \mathrm{which} \mathrm{is}$

11 similar to that of trion for transition $\sim 2.2 \mathrm{ps}$.

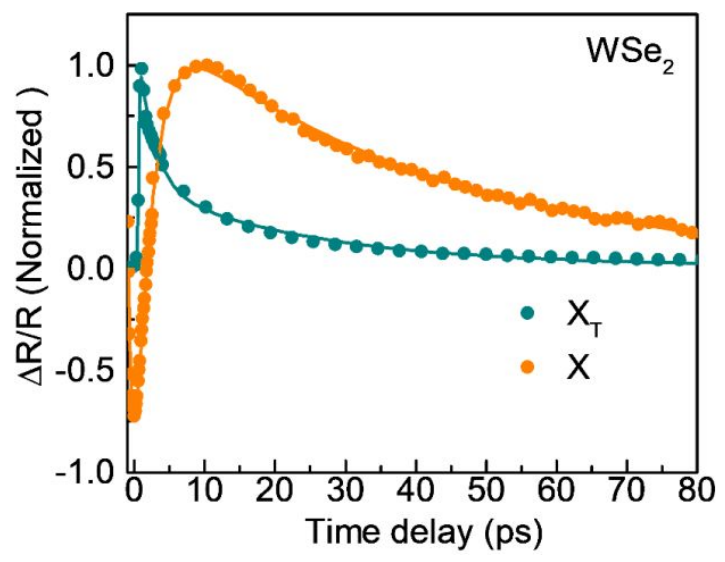

Figure S10. Differential reflection signal measured from monolayer $\mathrm{WSe}_{2}$ where the wavelength of pump pulse is fixed at $580 \mathrm{~nm}$. The orange and green solid lines indicate a bi-exponential decay fit of neutral exciton and trion, respectively. 
$1 \quad$ Table S1

\begin{tabular}{ccccc}
\hline $\mathrm{WSe}_{2}$ & $\mathrm{~A}_{1}$ & $\tau_{1}(\mathrm{ps})$ & $\mathrm{A}_{2}$ & $\tau_{2}(\mathrm{ps})$ \\
\hline $\mathrm{X}$ & 0.66 & 3.2 & 0.34 & 35.2 \\
$\mathrm{X}^{\mathrm{T}}$ & 0.6 & 2.2 & 0.4 & 23 \\
\hline
\end{tabular}

3 
2 Here we obtained the exciton related decay process in a short timescale within 3 ps by 3 subtracted from the baseline of the dotted line from the rising edge of the inset image in Figure

$4 \quad 4 \mathbf{f}$ and normalized to obtain the data as shown in Figure S11-1. We compared the exciton 5 related data with the fast decay process of the trions obtained from the inset image of Fiugre 3f

6 as shown in green solid circle. As can be seen from Fiugre 3f, the half-height width of both

7 excitons ang trions are quite similar. We have reason to speculate that this indicates to a certain

8 extent that the formation and relaxation process of the trions affect the time of the rising edge

9 of neutral excitons and the formation probability of neutral excitons in heterostructure with

10 twisted angle of $60^{\circ}$.

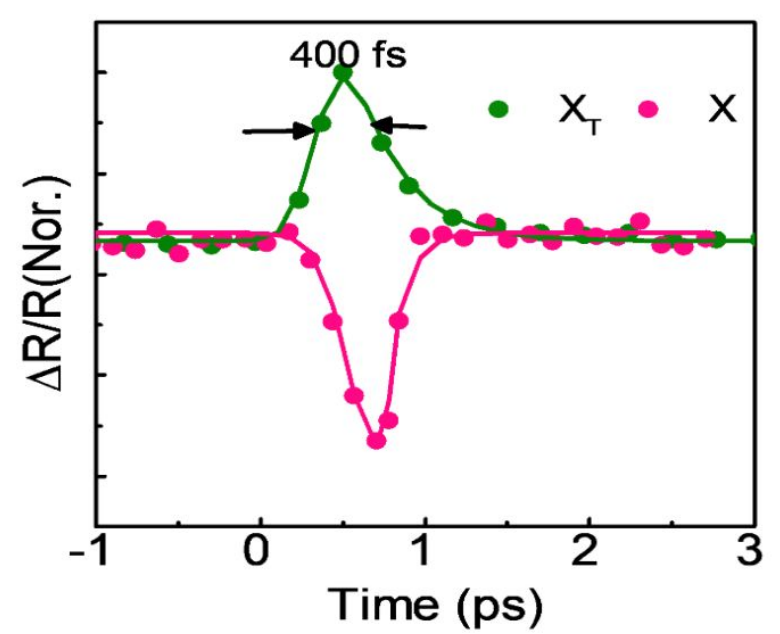

Figure S11-1. Differential reflection signal measured from $\mathrm{WS}_{2} / \mathrm{WSe}_{2}$ with twisted angle of $60^{\circ}$ where the wavelength of pump pulse is fixed at $580 \mathrm{~nm}$. The pink and green solid lines indicate a exponential decay fit of neutral exciton and trion, respectively. The pink solid circle is the data obtained by subtracting the baseline of the dotted line from the fast decay process with 3 ps of the inset image in figure $3 \mathbf{f}$ and the data is normalized. The half-height width is about $400 \mathrm{fs}$.

Here, the PL emission efficiency of the trions in heterostructure is both determined by the formation probability and formation time. As can be seen from Figure S11, the initial populations of excitons are larger than that of trions in heterostructure with twisted angle of $0^{\circ}$ 
and $60^{\circ}$, respectively. The excitons and trions share similar initial population in heterostructure with twisted angle $30^{\circ}$. For the heterostructure with twisted angle of $0^{\circ}$, the initial population of excitons is larger than that of trions while part of excitons are then transformed into trions owing to the redundant carriers. Besides the formation time and lifetime of excitons and trions are, excitons are comparable but excitons are formed in preference to the trions after optical pumping. The excitons are quickly formed within $\sim 1 \mathrm{ps}$ and converted into trions after $\sim 0.5 \mathrm{ps}$ as shown in Figure 3e in the manuscript. As a result, the trions exhibit a dominant luminescence in the eterostructure with twisted angle of $30^{\circ}$. For the heterostructure with twisted angle of $60^{\circ}$, the initial population of excitons is slightly larger than that of trions, while the formation time of trions is significantly faster than that of the excitons. We can observe a small concave signal on the rising edge of the excitons and the time scales of trions and the concave signal are comparable as shown in Figure S11-2. It indicates that excitons tend to preferentially form trions firstly, and then form excitons after relaxation of trions is completed and the formation process of the trions affects the formation of excitons. As a result, the higher PL emission efficiency in heterostructure with twisted angle of $60^{\circ}$ is mainly caused by the advantage of formation process.
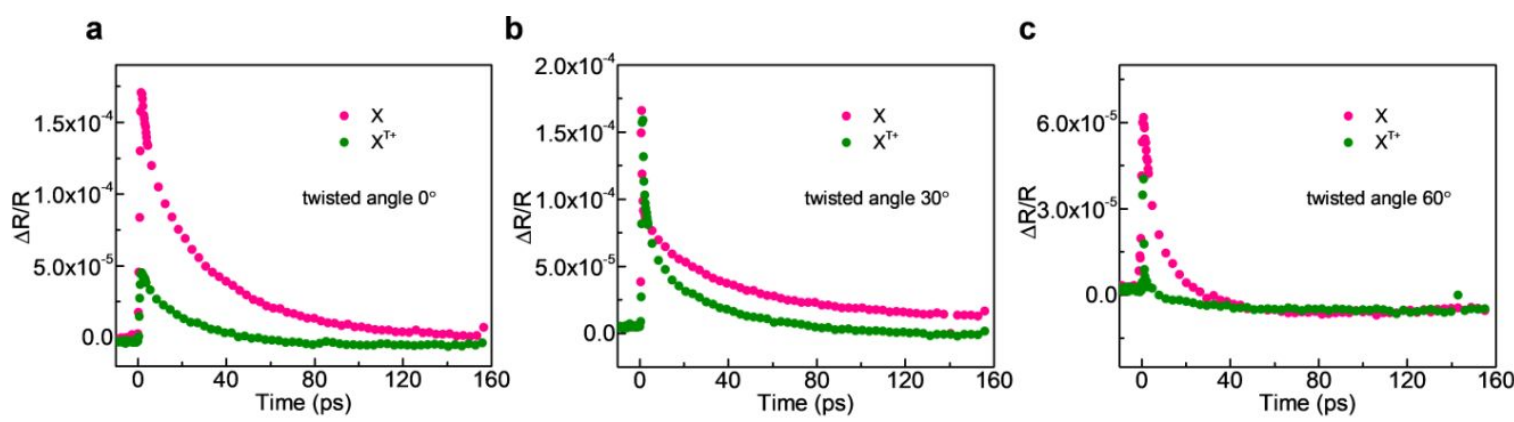

Figure S11-2. (a)-(c) Differential reflection signal measured from $\mathrm{WS}_{2} / \mathrm{WSe}_{2}$ heterostructure with twisted angles of $0^{\circ}, 30^{\circ}$, and $60^{\circ}$, respectively. 
The mass action law can be expressed as ${ }^{6-7}$

$$
\frac{\mathrm{N}_{\mathrm{A}} \mathrm{n}_{\mathrm{h}}}{\mathrm{N}_{\mathrm{A}^{+}}}=\frac{4 \mathrm{~m}_{\mathrm{A}} \mathrm{m}_{\mathrm{h}} \mathrm{k}_{\mathrm{B}} \mathrm{T}}{\pi \hbar^{2} \mathrm{~m}_{\mathrm{A}^{+}}} \exp \left(-\frac{\mathrm{E}_{\mathrm{A}^{+}}}{\mathrm{k}_{\mathrm{B}} \mathrm{T}}\right),
$$

$$
\frac{\mathrm{I}_{\mathrm{A}^{+}}}{\mathrm{I}_{\mathrm{A}}}=\frac{\Gamma_{\mathrm{A}^{+}}}{\Gamma_{\mathrm{A}}} \cdot \frac{\mathrm{N}_{\mathrm{A}^{+}}}{\mathrm{N}_{\mathrm{A}}},
$$

where $N_{A}$ and $N_{A}+$ represent the numbers of intralayer excitons $A$ and positive trions $A^{+} ; n_{h}$ is the number of holes; $m_{A}=m_{e}+m_{h}$ and $m_{A^{+}}=m_{e}+2 m_{h}$, where $m_{A}, m_{A^{+}}, m_{e}$, and $m_{h}$ represent the mass of an exciton $A$, a trion $A^{+}$, an electron, and a hole, respectively; $k_{\mathrm{B}}, T$, and $\hbar$ express Boltzmann's constant, temperature, and the reduced Planck's constant, respectively; $E_{A^{+}}$is the binding energy for the trion; and $\Gamma_{A^{+}}$and $\Gamma_{A}$ serve as the emission rates for trions and excitons, which can be obtained by the transient absorption spectrum above. Here, $m_{A}=0.79$ $m_{0}, m_{A^{+}}=1.25 m_{0}, m_{h}=0.46 m_{0}, m_{e}=0.33 m_{0}$ and $k_{B} T=25.9 \mathrm{meV}$, respectively. ${ }^{8}$ As can be deduced from the equation (1) and (2), the steady state holes concentration is proportional to $I_{A}^{+} / I_{A}$ and is inversely proportional to the $\Gamma_{A}+/ \Gamma_{A}$, which means $n_{h}$ is proportional to the intensity ratio of trions and excitons but inversely proportional to the lifetime ratio of trions and excitons. Here, the PL intensity ratio and lifetime ratio are both related to the twisted angle of the heterostructure, the key parameters that determine the hole concentration are angle dependent. For the heterostructure with twisted angle of $0^{\circ}$, both the value of $I_{A}+/ I_{A}$ and $\Gamma_{A^{+}} /$ $\Gamma_{A}$ are close to 1 . For the heterostructure with twisted angle of $30^{\circ}$, the value of $I_{A^{+}} / I_{A}$ is larger than 1 while the $\Gamma_{A}+/ \Gamma_{A}$ is close to 1 . As a result, the hole concentration in the heterostructure with twisted angle of $30^{\circ}$ is higher. For the heterostructure with twisted angle of $60^{\circ}$, the ratio value of $I_{A}+/ I_{A}$ and $\Gamma_{A}+/ \Gamma_{A}$ are about 5. As mentioned above, $n_{h}$ is proportional to the intensity ratio of trions and excitons but inversely proportional to the lifetime ratio of trions and excitons. Therefore, the hole cncentrations in heterostructure with twisted angle of $0^{\circ}$ and $60^{\circ}$ are in the same magtitude of order. As a result, the concentrations of holes in steady states of the heterostructure with twisted angles of $0^{\circ}, 30^{\circ}$, and $60^{\circ}$ are calculated to be $\sim 10^{12}, 10^{13}$, and $10^{12} \mathrm{~cm}^{-2}$, respectively. Through the calculation, we know that hole concentrations in different twisted angle heterostructures are within an order of magnitude 
1 for heterostructures with twisted angle of $0^{\circ}$ and $60^{\circ}$, which means that carrier concentration is

2 not the reason for the different ratios of trion luminescence. However, for heterostructure with

3 twisted angle of $30^{\circ}$, a large concentration of carrier density is achieved by hole transfer from

$4 \mathrm{WS}_{2}$ to $\mathrm{WSe}_{2}$, which leads to a large binding energy of trions in heterostructure with twisted

5 angle of $30^{\circ}$. For bilayer $\mathrm{MoS}_{2}$ with $\mathrm{AA}$ and $\mathrm{AB}$ stacking sequences, the interlayer interaction

6 leads the formation of intralayer trions and indirect excitons. Different from the heterostructure,

7 the indirect excitons transition occurs between the conduction band minimum halfway on the

$8 \mathrm{~K}-\Gamma$ line and the valence band maximum at the $\Gamma$ point. These states near these band extrema

9 originate from linear combinations of Mo-d and S-p orbitals and indirect excitons between the

10 layers does not participate in the formation of the trions in K-valley of monolayer materials. As

11 a result, the distributions of trions and its binding energy are not applicable to the K-K valley

12 transition (interlayer excitons, intralayer excitons and intralayer trions) in heterostructures. For

13 the heterostructure with twisted angle of $30^{\circ}$, a large concentration of carrier density $\left(10^{13} \mathrm{~cm}^{-2}\right)$

14 is achieved by hole transfer from $\mathrm{WS}_{2}$ to $\mathrm{WSe}_{2}$, which leads to a large binding energy of trions.

15 As for the interlayer excitons, it does not satisfy the momentum matching condition, so most of

16 the transferred holes are used to form intralayer trions. 
2 1. Lui, C. H.; Ye, Z.; Ji, C.; Chiu, K.-C.; Chou, C.-T.; Andersen, T. I.; Means-Shively, C.; 3 Anderson, H.; Wu, J.-M.; Kidd, T.; Lee, Y.-H.; He, R., Observation of Interlayer Phonon 4 Modes in Van Der Waals Heterostructures. Phys. Rev. B 2015, 91, 165403.

5 2. Nayak, P. K.; Horbatenko, Y.; Ahn, S.; Kim, G.; Lee, J. U.; Ma, K. Y.; Jang, A. R.; Lim, 6 H.; Kim, D.; Ryu, S.; Cheong, H.; Park, N.; Shin, H. S., Probing Evolution of 7 Twist-Angle-Dependent Interlayer Excitons in $\mathrm{MoSe}_{2} / \mathrm{WSe}_{2}$ Van Der Waals Heterostructures. 8 ACS nano 2017, 11, 4041.

9 3. van der Zande, A. M.; Kunstmann, J.; Chernikov, A.; Chenet, D. A.; You, Y.; Zhang, X.; 10 Huang, P. Y.; Berkelbach, T. C.; Wang, L.; Zhang, F.; Hybertsen, M. S.; Muller, D. A.; 11 Reichman, D. R.; Heinz, T. F.; Hone, J. C., Tailoring the Electronic Structure in Bilayer 12 Molybdenum Disulfide Via Interlayer Twist. Nano Lett. 2014, 14, 3869.

13 4. Hu, X.; Kou, L.; Sun, L., Stacking Orders Induced Direct Band Gap in Bilayer $14 \mathrm{MoSe}_{2}-\mathrm{WSe}_{2}$ Lateral Heterostructures. Sci. Rep. 2016, 6, 31122.

15 5. Hsu, W.-T.; Lu, L.-S.; Wu, P.-H.; Lee, M.-H.; Chen, P.-J.; Wu, P.-Y.; Chou, Y.-C.; Jeng, 16 H.-T.; Li, L.-J.; Chu, M.-W.; Chang, W.-H., Negative Circular Polarization Emissions from $17 \mathrm{WSe}_{2} / \mathrm{MoSe}_{2}$ Commensurate Heterobilayers. Nat. Commun. 2018, 9, 1356.

18 6. Lin, Y.; Ling, X.; Yu, L.; Huang, S.; Hsu, A. L.; Lee, Y.-H.; Kong, J.; Dresselhaus, M. S.; 19 Palacios, T., Dielectric Screening of Excitons and Trions in Single-Layer $\mathrm{MoS}_{2}$. Nano Lett. 20 2014, 14, 5569.

21 7. Zhang, Q.; Naylor, C. H.; Gao, Z.; Wu, R.; Abidi, I. H.; Zhao, M. Q.; Ding, Y.; Cagang, 22 A. A.; Zhuang, M.; Ou, X.; Luo, Z., Recoil Effect and Photoemission Splitting of Trions in 23 Monolayer $\mathrm{MoS}_{2}$. ACS nano 2017, 11, 10808.

24 8. Jadczak, J.; Kutrowska-Girzycka, J.; Kapuscinski, P.; Huang, Y. S.; Wojs, A.; Bryja, L., 25 Probing of Free and Localized Excitons and Trions in Atomically Thin $\mathrm{WSe}_{2}, \mathrm{WS}_{2}, \mathrm{MoSe}_{2}$ and $\mathrm{MoS}_{2}$ in Photoluminescence and Reflectivity Experiments. Nanotechnology 2017, 28, 395702. 